\title{
Evaluation of the Circles Measurement and the ABC Classification of Acromioclavicular Joint Injuries
}

\author{
Richard J. Murphy, ${ }^{\star \dagger \ddagger \S ~ M B C h B, ~ M A, ~ D P h i l, ~ B e a t ~ K . ~ M o o r, " ~ M D, ~ P i o t r ~ J . ~ L e s n i e w s k i, ~}{ }^{\dagger \ddagger ~ M D, ~}$ \\ Annabel Hayoz, ${ }^{\dagger}$ MSc, Wolfan Alcantara, ${ }^{\dagger} \mathrm{MD}$, and Matthias A. Zumstein, ${ }^{\star \dagger \neq \uparrow ~ M D ~}$ \\ Investigation performed at Inselspital, Bern, Switzerland, and Sonnenhof Orthopaedics, \\ Bern, Switzerland
}

\begin{abstract}
Background: Acromioclavicular joint (ACJ) injuries are common. Despite this, it remains unclear how best to assess, classify, and manage these cases. A simple, reliable, valid, and accurate radiographic parameter to measure ACJ displacement would allow improved consistency of diagnosis and subsequent treatment pathways.
\end{abstract}

Purpose: To evaluate "the circles measurement" and associated "ABC classification" as a tool for assessing ACJ displacement and injury classification.

Study Design: Descriptive laboratory study.

Methods: The circles measurement is taken from a lateral Alexander radiograph of the shoulder. The measurement is the centerto-center distance between 2 circles drawn to define the lateral extent of the clavicle and the anteromedial extent of the acromion; it is independent of the displacement plane, judging total ACJ displacement in any direction rather than trying to quantify vertical and/or horizontal displacement. When utilized clinically, the circles measurement is a single measurement calculated as the difference between values recorded for the injured and uninjured sides. Validation of the circles measurement was performed using lateral Alexander radiographs (including $\pm 20^{\circ}$ projection error in all planes) and computed tomography of standardized ACJ injury simulations. We assessed inter- and intrarater reliability, convergent validity, and discriminant validity of the circles measurement and subsequently generated a classification of ACJ injury based on displacement.

Results: Reliability and validity of the circles measurement was excellent throughout. Interrater reliability (ICC [intraclass correlation coefficient] [2,1], 95\% Cl; $n=78 ; 4$ observers) was 0.976 (0.964-0.985). Intrarater reliability (ICC [2,1]; 95\% Cl; $n=78 ; 2$ measures) was 0.998 (0.996-0.998). Convergent validity (Pearson correlation coefficient, $r$ ) was 0.970 for ideal radiographs and 0.889 with $\pm 20^{\circ}$ projection error in all planes. Discriminant validity, with 1 -way analysis of variance, showed a $P$ value of $<.0001$ and effect size $\left(\eta^{2}\right)$ of 0.960 , with the ability to distinguish between the previously defined stable (Rockwood IIIA) and unstable (Rockwood IIIB) injuries. The results permitted objective, statistically sound parameters for the proposed ABC classification system.

Conclusion: The circles measurement is a simple, reliable, valid, accurate, and resilient parameter for assessing ACJ displacement and can be used in conjunction with the proposed $A B C$ classification to define ACJ injuries more accurately and objectively than previously described.

Clinical Relevance: This novel parameter has the potential to standardize the initial assessment and possibly the subsequent clinical management of ACJ injuries, in addition to providing a standardized measure for future research.

Keywords: general sports trauma; shoulder; acromioclavicular joint; imaging and radiology; general; clinical assessment/grading scales

Injuries to the acromioclavicular joint (ACJ) are common, occurring in $9 \%$ of shoulder traumas ${ }^{16}$; despite this, controversy remains over their classification, imaging, and subsequent management. ${ }^{3,4,15,21,26,29}$ The most commonly used

The American Journal of Sports Medicine

2021;49(6):1619-1625

DOI: $10.1177 / 03635465211003300$

(c) 2021 The Author(s) classification remains the Rockwood radiological interpretation of the Tossy classification (1963), despite its lack of high interrater reliability. ${ }^{5,21,23,28}$ Quantitative parameters of superoinferior, or vertical, displacement include the difference in coracoacromial distance between injured and uninjured sides on panoramic large cassette radiographs, although this is prone to significant variability with projection error and little appreciation of anteroposterior or horizontal displacement. ${ }^{5,8,23,30}$ Attempts to assess horizontal displacement include axillary radiographic projections, 
although research suggests these views can be misleading. ${ }^{22,27}$ The Alexander view, ${ }^{1}$ a lateral stress radiograph taken with the hand of the arm being imaged placed in the contralateral axilla, facilitates evaluation of horizontal and vertical displacement on a single view. ${ }^{10,18,31}$ Figure 1 shows a large cassette panoramic view, with comparative Alexander views from the same individual, illustrating the utility of the Alexander view in demonstrating both vertical and horizontal displacement. Figure 2 shows the method for obtaining an Alexander view radiograph in a clinical setting. The acromial center line to dorsal clavicle measurement demonstrates good quantification of ACJ displacement, although it is complicated, time-consuming, and prone to error without adequate training and experience. ${ }^{31}$ Other radiological modalities, such as magnetic resonance imaging and computed tomography (CT), demonstrate little additional benefit, but a significant increase in costs, time, and risk of inflating the extent of the injury. ${ }^{2,20,24}$

The original classification ${ }^{28}$ describes injury to the 3 ACJ ligamentous constraints: joint capsule, conoid ligament, and trapezoid ligament. Biomechanical studies show that the joint capsule confers horizontal stability and fails first during injury, followed by the conoid and trapezoid ligaments, which confer further horizontal stability, although mainly acting as restraints to vertical displacement. $6,7,9,11,12,14,17,19$ A lateral-to-medial mechanism of injury would, therefore, cause horizontal instability before vertical instability, a concept that questions the underlying premise of the Tossy classification. ${ }^{28}$ The positions of clavicular displacement described by Tossy remain useful, even if the underlying ligamentous injury pattern may be questioned.

Clinical management of ACJ injuries also remains controversial, particularly Rockwood III injuries. ${ }^{4,15,26,29} \mathrm{~A}$ distinction between stable and unstable injuries, included in the International Society of Arthroscopy, Knee Surgery and Orthopaedic Sports Medicine (ISAKOS) consensus statement on management of ACJ injuries, is based on the presence (IIIB) or absence (IIIA) of horizontal instability. ${ }^{4}$ Even with this distinction, however, the classification and subsequent treatment pathway for patients with ACJ injuries is unclear and subject to significant variation.

Such uncertainty in classification, vector of displacement, and imaging choice, combined with complexity and unreliability of radiological measurements before a clinical decision is taken, calls for a simple, quick, reliable means of quantifiable radiological assessment to inform specialist

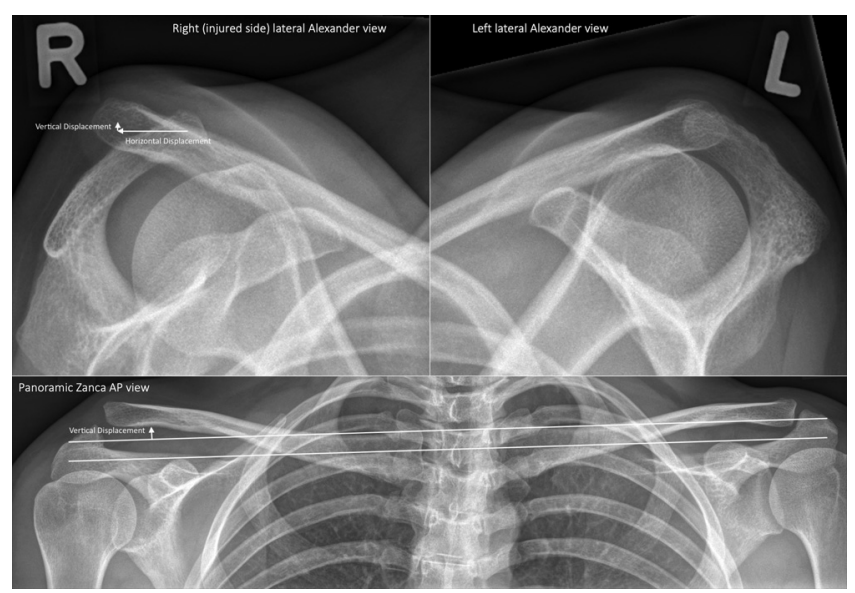

Figure 1. Radiographs from 1 individual on the day of injury to the right acromioclavicular joint. The top images show lateral Alexander views of each side and demonstrate the visualization of vertical and, particularly, horizontal displacement. The lower large cassette panoramic radiograph below demonstrates how this view does not provide any indication of horizontal instability in such cases. AP, anteroposterior.

practice, standardize research, and guide the nonspecialist when triaging these injuries.

This study aimed to evaluate the reliability, validity, and accuracy of a novel radiological parameter, the circles measurement, in objectively evaluating ACJ displacement and developing an associated classification. We hypothesized that the circles measurement would show adequate convergent and discriminant validity in serving as a reliable, objective measure of ACJ displacement.

\section{METHODS}

This diagnostic study used radiographs of Sawbones models for all validation work; ethical approval was not required.

\section{Radiographic Images}

We used standardized radiographs and CT images taken from a previously described ACJ injury Sawbones model. ${ }^{31}$ A specially designed jig, incorporating a radiopaque

*Address correspondences to Matthias A. Zumstein, MD, Shoulder, Elbow and Orthopaedic Sports Medicine, Sonnenhof Orthopaedics, Salvisbergstrasse 4, Bern, 3006, Switzerland (email: m.zumstein@me.com); Richard J. Murphy, MBChB, MA, DPhil, University Hospitals Sussex NHS Foundation Trust, Royal Sussex County Hospital, Eastern Road, Brighton, BN2 5BE, UK (email: richardjamesmurphy@icloud.com) (Twitter: @Dr_Rich_Murphy).

'Shoulder, Elbow and Orthopaedic Sports Medicine, Sonnenhof Orthopaedics, Bern, Switzerland.

${ }^{\ddagger}$ Shoulder, Elbow and Orthopaedic Sports Medicine, Department of Orthopaedic Surgery and Traumatology, Inselspital, Bern University Hospital, Bern, Switzerland.

§University Hospitals Sussex NHS Foundation Trust, Royal Sussex County Hospital, Brighton, UK.

"Hôpital du Valais (RSV) - Centre Hospitalier du Valais Romand, Hôpital de Martigny, Martigny, France.

"Stiftung Lindenhof I Campus SLB, Swiss Institute for Translational and Entrepreneurial Medicine, Bern, Switzerland.

Submitted July 2, 2020; accepted December 22, 2020.

One or more of the authors has declared the following potential conflict of interest or source of funding: This study was supported by an EFORT fellowship grant (R.J.M. and P.J.L.). M.A.Z. has received consulting fees from Medacta International. AOSSM checks author disclosures against the Open Payments Database (OPD). AOSSM has not conducted an independent investigation on the OPD and disclaims any liability or responsibility relating thereto. 


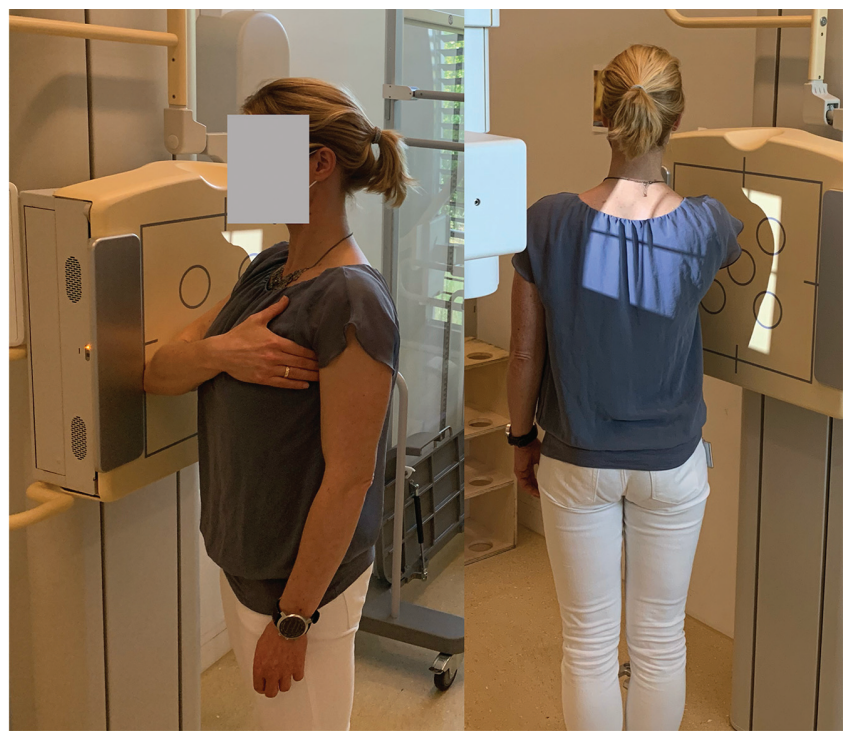

Figure 2. Photographs of a member of the research team positioned for a lateral Alexander view projection of the right shoulder. The right hand placed in the left axilla and a radiograph taken for lateral scapular view.

calibration ball, held the Sawbones in place during simulation of the following ACJ injuries: normal/uninjured, Rockwood II, Rockwood IIIA (isolated vertical displacement), Rockwood IIIB (vertical and horizontal displacement), Rockwood IV, and Rockwood V., ${ }^{43}$ Parameters of displacement were in keeping with the previously published model (Table 1). ${ }^{31}$ For each injury group, we acquired 13 lateral radiographs plus a 3-dimensional (3D) CT reconstruction; the radiographs simulated an Alexander view. ${ }^{1}$ The 13 radiographs taken for each of the 6 injury models included a perfect projection, the neutral image, and 12 further images showing rotational projection error of $+10^{\circ},-10^{\circ},+20^{\circ}$, and $-20^{\circ}$ in each of the 3 anatomic planes, respectively. Thus, we acquired a total of 78 radiographs across the 6 injury groups; we evaluated the radiographs and $63 \mathrm{D} \mathrm{CT}$ reconstructions using a picture archiving and communications system (PACS, SECTRA AB).

\section{Radiographic Parameters}

We performed circles measurements on lateral Alexander projections of the shoulder girdle as shown in Figure 3; each observer drew all annotations from a plain image to generate their measurements. We recorded all values to the nearest $0.1 \mathrm{~mm}$. A clinically relevant parameter was calculated as the difference between the injured and uninjured sides.

An independent radiologist calculated displacement of the ACJ as the magnitude of the vector of total displacement on CT reconstructions, recorded to the nearest $0.1 \mathrm{~mm}$. This measurement stood as the gold standard for ACJ displacement.
TABLE 1

Magnitude of ACJ Displacement for Each Simulated Injury Group in the ACJ Injury Sawbones $\mathrm{Model}^{a}$

\begin{tabular}{lcc}
\hline $\begin{array}{l}\text { Injury Simulation } \\
\text { Group }\end{array}$ & $\begin{array}{c}\text { Vertical } \\
\text { Displacement (\%) }\end{array}$ & $\begin{array}{c}\text { Horizontal } \\
\text { Displacement (\%) }\end{array}$ \\
\hline Control (uninjured) & 0 & 0 \\
Rockwood II & 50 & 0 \\
Rockwood IIIA & 100 & 0 \\
Rockwood IIIB & 100 & 50 \\
Rockwood IV & 200 & 100 \\
Rockwood V & 200 & 200 \\
\hline
\end{tabular}

${ }^{a}$ Vertical displacement indicates superior movement of the distal clavicle relative to the acromion, and horizontal displacement indicates posterior movement of the distal clavicle relative to the acromion. ACJ, acromioclavicular joint.
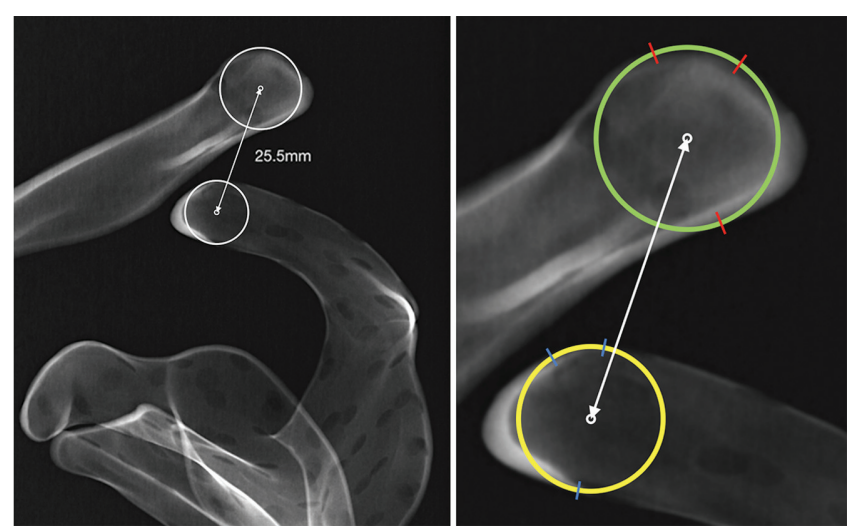

Figure 3. Circles measurement of acromioclavicular joint (ACJ) displacement. The left-hand image shows how the measurement looks in the picture archiving and communications system; these are all the radiographic annotations required in clinical use. The right-hand image shows a magnification with graphical features of the measurement for descriptive purposes. The method is as follows: first, draw 2 circles: 1 defining the lateral extent of the clavicle (green circle), its circumference contacting the outer edge of the superior, inferior, and posterolateral cortices of the clavicle, without exiting the bone shadow (red dashes); and a second circle defining the anteromedial extent of the acromion (yellow circle), with its circumference contacting the outer edge of the superior, inferior, and anteromedial cortices of the acromion, without exiting the bone shadow (blue dashes). Measure the center-to-center distance between the 2 circles (white arrowed line between the 2 white circles); this is the absolute circles measurement for a single side. Clinically, the circles measurement is a single measurement generated by taking the difference between injured and uninjured sides.

\section{Intra- and Interobserver Reliability}

Four assessors, each blinded to the others' measurements, calculated ACJ displacement using the circles measurement on the 78 radiographic images from the 6 Sawbones 
injury groups. The assessors included 2 fellowship-trained shoulder and elbow surgeons (R.J.M. and P.J.L.), an orthopaedic resident with minimal experience in shoulder surgery (W.A.), and a research coordinator with no previous experience in shoulder surgery or radiographic measurements (A.H.). Each assessor received simple instructions on the circles measurements as detailed in Figure 3. Assessors without previous experience of using PACS software received guidance on navigating PACS, drawing circles, and measuring a distance on an image; they received no other training.

We took measurements in a random image order and recorded the results separately to the nearest $0.1 \mathrm{~mm}$ before comparing the results to assess interobserver reliability. One assessor (P.J.L.) completed measurements on 2 separate occasions, 2 weeks apart.

\section{Statistical Analysis}

We recorded all individual measurements to $0.1 \mathrm{~mm}$ and presented results as means with standard deviation or 95\% CI as indicated. We assessed normality using a KolmogorovSmirnoff test before parametric testing.

Reliability and convergent validity statistics for the circles measurement used individual absolute values from single images; this process allowed for assessment of the reproducibility of each measurement taken. In contrast, for all diagnostic injury group comparisons and clinical assessments, we used the mean observer values for the difference in circles measurement between injured and uninjured sides, as would be the case when such a measurement is used in clinical practice.

A previous power calculation suggested a minimum of 3 assessors and 33 comparative measurements to determine intra- and interobserver reliability with $80 \%$ power and .05 significance level. ${ }^{31}$ For completeness and to evaluate the effect of using uninitiated observers, we employed 4 assessors, each taking measurements from all 78 Sawbones radiographs. We calculated intra- and interobserver reliability using a 2-way random intraclass correlation coefficient (ICC), assuming single measurement and absolute agreement (ICC $[2,1])$, and presented with a $95 \%$ CI. ${ }^{25}$ We interpreted the results according to $\mathrm{Koo}$ and $\mathrm{Li}^{13}$ $(<0.5=$ poor; $0.5-0.75=$ moderate $;>0.75-0.9=$ good ; and $>0.90=$ excellent reliability).

We assessed convergent validity of the circles measurement against displacement measured on 3D CT with calculation of Pearson correlation coefficient $(r)$ for neutral images $(\mathrm{n}=6)$, neutral $\pm 10^{\circ}$ projection error in all planes $(\mathrm{n}=42)$, and $\pm 20^{\circ}$ projection error in all planes $(\mathrm{n}=78)$. We categorized the degree of correlation as $<0.2=$ poor, 0.2-0.4 = low, $>0.4-0.6=$ moderate, $>0.6-0.8=$ good, and greater than $0.8=$ excellent. We performed a regression analysis of circles versus CT-measured displacement.

Using data from the Sawbones model radiographs, we applied an independent 1-way analysis of variance (ANOVA) test to assess discriminant validity of the circles measurements for injury diagnosis (ie, difference between injured and uninjured sides) and calculated the effect
TABLE 2

Convergent Validity for Absolute Circles Measurements ${ }^{a}$

\begin{tabular}{lcr}
\hline Convergence & $r$ & $P$ Value \\
\hline Neutral $(\mathrm{n}=6)$ & 0.970 & .0013 \\
$\pm 10 \%(\mathrm{n}=42)$ & 0.933 & $<.0001$ \\
$\pm 20 \%(\mathrm{n}=78)$ & 0.889 & $<.0001$ \\
\hline
\end{tabular}

${ }^{a}$ Convergent validity for absolute circles measurements shown as correlation with acromioclavicular joint displacement measure on 3-dimensional computed tomography. Pearson correlation coefficients $(r)$ with $95 \%$ CI for perfect neutral radiographs and with $\pm 10 \%$ or $\pm 20 \%$ projection error.

size $\left(\eta^{2}\right)$; we performed multiple intergroup comparisons, including a Bonferroni correction. Discriminant validity and intergroup comparisons evaluated the ability of the circles measurement to distinguish between injury groups.

We performed statistical analyses using $\mathrm{R}$, a language and environment for statistical computing ( $\mathrm{R}$ Core Team, 2016; $\mathrm{R}$ Foundation for Statistical Computing) and GraphPad Prism Version 8.3.1 for Mac OS (GraphPad Software Inc).

\section{RESULTS}

\section{Reliability and Convergent Validity}

Inter- and intraobserver reliability for the circles measurement was excellent in all comparisons. Interrater reliability (ICC [2,1]; 95\% CI; $\mathrm{n}=78 ; 4$ observers) was 0.976 (0.964-0.985), and intrarater reliability (ICC $[2,1] ; 95 \%$ $\mathrm{CI} ; \mathrm{n}=78$; 2 measures) was 0.998 (0.996-0.998).

The circles measurement showed excellent convergent validity when compared with CT- measured displacement (Table 2). Regression analysis results of CT-measured displacement versus the circles measurement showed a slope of $0.889, y$-intercept of $1.4 \mathrm{~mm}$ in favor of the circles measurement, and a goodness-of-fit $R^{2}$ value of 0.776 , which is extremely high, particularly as it allows for $\pm 20^{\circ}$ projection error in all planes. In general terms, this means that the circles measurement quantifies the precise displacement of the ACJ with an extremely high degree of accuracy and remains accurate in the face of poor-quality radiographs, with error of projection of up to $20^{\circ}$ in any direction.

\section{Discriminant Validity and Injury Classification}

The circles measurement demonstrated excellent discriminant validity; results of 1 -way ANOVA showed a $P$ value of $<.0001$ and effect size $\left(\eta^{2}\right)$ of 0.960 . Intergroup analysis results revealed a significant difference in mean circles measurement for all adjacent injury groups (Table 3). This means that the circles measurement can consistently distinguish between each Rockwood grade of injury in an objective and reproducible manner.

The lower limit of the 95\% CI in the Rockwood IIIB group was $7.0 \mathrm{~mm}$ and the lower limit of the $95 \% \mathrm{CI}$ in the Rockwood IV group was $14.4 \mathrm{~mm}$. Figure 4 displays 
TABLE 3

Intergroup Analysis

Between Different Rockwood Groups ${ }^{a}$

\begin{tabular}{lcr}
\hline $\begin{array}{l}\text { Rockwood Group } \\
\text { Comparison }\end{array}$ & $\begin{array}{c}\text { Difference Between } \\
\text { Means }(\mathrm{mm})\end{array}$ & $P$ Value \\
\hline Normal vs II & 2.3 & .0184 \\
II vs IIIa & 2.5 & .0081 \\
IIIa vs IIIb & 4.2 & $<.0001$ \\
IIIb vs IV & 7.5 & $<.0001$ \\
IV vs V & 7.1 & $<.0001$ \\
\hline
\end{tabular}

${ }^{a}$ Intergroup analysis of means from 1-way analysis of variance for each of the adjacent injury groups, including Bonferroni correction of $P$ values for multiple comparisons. A significant difference in measurements was seen in each adjacent pair of injury groups, demonstrating the capacity of the circles measurement to differentiate between these injuries.

these results and the proposed $\mathrm{ABC}$ classification for ACJ displacement.

\section{DISCUSSION}

The management of ACJ injuries remains a topic of debate; a significant contributing factor to this disagreement is the lack of a reliable, objective means of classifying injuries. It is accepted that unstable injuries demonstrate the greatest displacement of the ACJ and also have the strongest indication for surgical stabilization, as described by the ISAKOS consensus guidelines. ${ }^{4}$ The decision-making process is complex and lacks any reproducible and easily applied objective measure, particularly radiological. Extensive biomechanical work has been published describing the effect of injury to each of the static restraints of the ACJ. ${ }^{6,7,9,11,12,14,17,19}$ In a clinical setting, however, it is not possible to accurately establish which specific ligaments are damaged, and knowing such detail may be of limited value in deciding on treatment. Ultimately, the magnitude of displacement of the ACJ in any direction represents the most significant objective measure of joint instability. An accurate and reliable means of measuring such displacement may provide a reproducible scale with utility in predicting subsequent prognosis and necessity for operative intervention.

The results of our study show the circles measurement to be a simple-to-use, reliable, valid, accurate, and resilient parameter for assessing ACJ displacement. The discriminant validity demonstrated by this novel parameter permitted development of a proposed ABC classification for ACJ injuries that may provide an objective means of standardizing future research and, ultimately, clinical practice.

The Rockwood classification and coracoclavicular distance are the most common parameters used clinically, although they do not show high interrater reliability and are subject to significant radiological projectional variation. ${ }^{5,21,23}$ The acromial center line to dorsal clavicle measurement provides an objective assessment of ACJ displacement but has limitations. It is complex and timeconsuming to perform and fails to show adequate

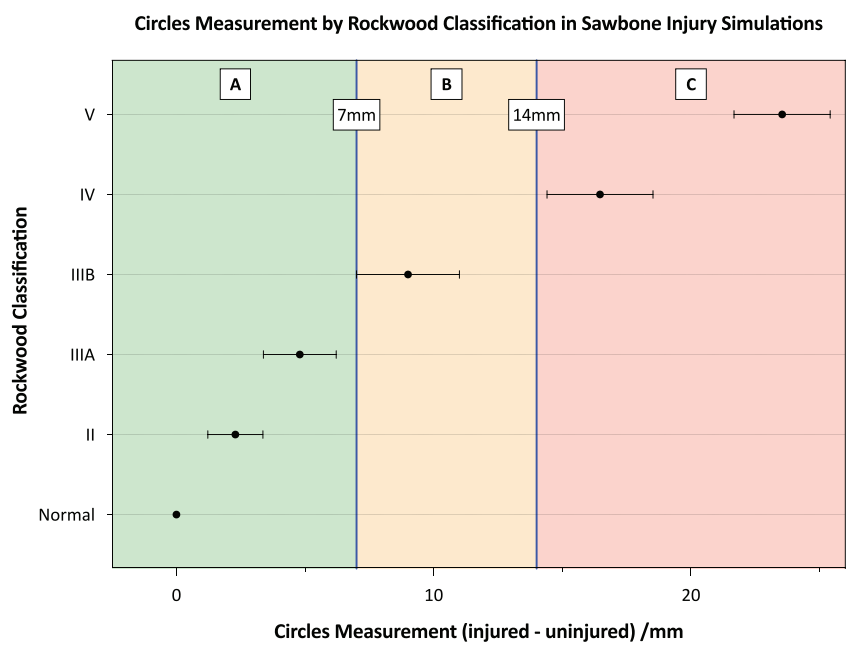

Figure 4. Graphical representation of average circles measurements (injured minus uninjured side) for each Sawbones injury group (error bars show 95\% Cl). The graph includes the proposed $A B C$ classification groups: $A=$ minimally displaced, less than $7 \mathrm{~mm}$ (equivalent to Rockwood IIIA or less); $\mathrm{B}=$ moderately displaced, 7 to $14 \mathrm{~mm}$ (equivalent to Rockwood IIIB); and C = significantly displaced, greater than $14 \mathrm{~mm}$ (equivalent to Rockwood IV and V).

discriminant validity to distinguish between Rockwood injury types IIIA and IIIB. ${ }^{10,31}$

The circles measurement was developed to address these limitations. The measurement is independent of displacement plane, judging total ACJ displacement in any direction rather than trying to quantify vertical and/or horizontal displacement. The Alexander view provides a stressed, single projection that shows displacement in both the vertical (coronal) and horizontal (axial) planes simultaneously, and so is ideal for this purpose. ${ }^{1}$ Considering ACJ displacement as a magnitude rather than a vector reduces error, variability, and complexity and ignores a parameter that has not been shown to be useful or reliable in clinical decision making (ie, component of displacement that is vertical and/or horizontal).

The circles measurement is reliable between raters of all levels of experience $(\mathrm{ICC}=0.976)$, from no experience of shoulder surgery or radiological assessment to fellowship-trained shoulder surgeons. As an absolute value, the circles measurement has excellent convergent validity when compared with CT-measured displacement of the ACJ; this validity remains excellent up to $\pm 20^{\circ}$ projectional error in all 3 anatomic planes. This means that the measurement is still accurate when assessed on highly suboptimal radiographs, as may be encountered in clinical practice. In practice, a radiograph that is malrotated by $20^{\circ}$ provides a highly abnormal view of the scapula and may result in reimaging in most cases. As such, the ability of this parameter to tolerate such a vast degree of radiographic malrotation makes it a highly useful tool, and clinicians adopting this technique can do so with confidence that a "perfect" Alexander view radiograph is not essential to the validity of the measurement. The accuracy 
of this parameter and its resilience to projectional error make it externally valid for clinical application, even in nonspecialist settings such as emergency and general radiology departments.

Ultimately, an objective measurement and injury classification should have the capacity to inform prognosis and treatment pathway. International consensus has split ACJ injuries into 3 groups for guidance of initial management: nonoperative (Rockwood types I to IIIA), operative (Rockwood types IV and greater), and an intermediate group who undergo an initial period of nonoperative treatment before further clinical assessment (Rockwood IIIB). ${ }^{4}$ One difficulty with this algorithm is the lack of reliability between clinicians in determining Rockwood classification. ${ }^{5,21,23,28}$ The circles measurement is able to define these groups extremely well and presents clear boundaries for objective assessment and introduction of our proposed $\mathrm{ABC}$ classification. Less than $7 \mathrm{~mm}$ difference in circles measurement between injured and uninjured sides denotes type A: minimally displaced, equivalent to Rockwood I, II, or IIIA, and suggested to be stable with an indication for nonoperative treatment according to the ISAKOS guidelines. Measurements from 7 to $14 \mathrm{~mm}$ denote type B: moderately displaced, equivalent to Rockwood IIIB, and potentially unstable according to international consensus, with a trial of nonoperative treatment. Measurements greater than 14 $\mathrm{mm}$ denote type $\mathrm{C}$ : significantly displaced, equivalent to Rockwood IV or greater, with a clear indication to offer or at least consider prompt surgical treatment. ${ }^{4}$ Despite this guidance, we emphasize that all clinical decisions should be taken in a holistic manner and liaison with each patient, giving full consideration to his or her medical condition, functional level, and personal wishes. To improve standardization of assessment and initial treatment recommendations, however, we propose considering modification of the ISAKOS algorithm to use the circles measurement as an objective parameter to determine injury group, rather than Rockwood classification, in conjunction with clinical assessment and patient choice.

The reliability, validity, and resilience to projectional error of the circles measurement and $\mathrm{ABC}$ classification support their application in specialist centers but also by nonspecialists, such as emergency department doctors, nurses, or physical therapists without the need for specialist involvement at the initial injury stage. In large, busy health care settings, such a simple, quick, objective, and accurate measure could be used to more effectively triage patients with ACJ injuries to the most appropriate pathway and may also lead to cost savings.

Limitations of this study include the in vitro nature of the validation: the setup, Sawbones models, and radiographic technique were all standardized and may not be applicable in all areas of clinical practice. The simulated injury scenarios were based on the descriptive Rockwood classification, made objective with the Sawbones constructs, which may not reflect genuine in vivo injury patterns, although they do provide an accurate and stepwise measure of displacement for validation and reflect existing worldwide consensus on ACJ injury patterns. While the circles measurement and $\mathrm{ABC}$ classification are not designed to be used in isolation, they can serve as a valuable adjunct to clinical assessment and patient consultation. Future use of both the circles measurement and the $\mathrm{ABC}$ classification in clinical practice and prospective research will serve to further validate their application and utility. We do not recommend these tools replace clinical acumen and adequate patient counseling; however, they represent, for the first time, an opportunity to standardize objective ACJ assessment. These tools can be used to compare results from different centers and provide an outcome measure that would support a collaborative multicenter analysis of assessment, treatment options, and outcomes after ACJ injury.

\section{CONCLUSION}

The circles measurement and $\mathrm{ABC}$ classification provide a simple, reliable, valid, accurate, and resilient parameter for assessing ACJ displacement. We believe that these parameters can be utilized in clinical and research settings to standardize the classification of ACJ injuries objectively to increase the validity of clinical research in this area and improve clinical outcomes in the longer term.

\section{ACKNOWLEDGMENT}

The authors thank Martin H. Maurer, a radiologist at their department, for her assistance in performing the calculation of the displacement of the ACJ on CT reconstructions.

\section{REFERENCES}

1. Alexander OM. Dislocation of the acromioclavicular joint. Radiography. 1949;15(179):260.

2. Alyas F, Curtis M, Speed C, Saifuddin A, Connell D. Magnetic resonance imaging appearances of acromioclavicular joint dislocation. Radiographics. 2008;28(2):463-479.

3. Beitzel K, Cote MP, Apostolakos J, et al. Current concepts in the treatment of acromioclavicular joint dislocations. Arthroscopy. 2013;29(2):387-397.

4. Beitzel K, Mazzocca AD, Bak K, et al. International Society of Arthroscopy, Knee Surgery, and Orthopaedic Sports Medicine (ISAKOS) upper extremity committee consensus statement on the need for diversification of the Rockwood classification for acromioclavicular joint injuries. Arthroscopy. 2014;30:271-278.

5. Cho C-H, Hwang I, Seo J-S, et al. Reliability of the classification and treatment of dislocations of the acromioclavicular joint. $J$ Shoulder Elbow Surg. 2014;23(5):665-670.

6. Debski RE, Parsons IM, Woo SL-Y, Fu FH. Effect of capsular injury on acromioclavicular joint mechanics. $J$ Bone Joint Surg Am. 2001;83(9):1344-1351.

7. Fukuda K, Craig EV, An KN, Cofield RH, Chao EY. Biomechanical study of the ligamentous system of the acromioclavicular joint. $J$ Bone Joint Surg. 1986;68(3):434-440.

8. Gastaud O, Raynier JL, Duparc F, et al. Reliability of radiographic measurements for acromioclavicular joint separations. Orthop Traumatol Surg Res. 2015;101(8)(suppl):S291-S295.

9. Harris RI, Wallace AL, Harper GD, Goldberg JA, Sonnabend DH, Walsh WR. Structural properties of the intact and the reconstructed coracoclavicular ligament complex. Am J Sports Med. 2000;28(1):103-108.

10. Karargyris O, Murphy RJ, Arenas A, Bolliger L, Zumstein MA. Improved identification of unstable acromioclavicular joint injuries in a clinical 
population using the acromial center line to dorsal clavicle radiographic measurement. J Shoulder Elbow Surg. 2020;29(8):1599-1605.

11. Keener JD. Acromioclavicular joint anatomy and biomechanics. Oper Techn Sport Med. 2014;22(3):210-213.

12. Klimkiewicz JJ, Williams GR, Sher JS, Karduna A, Jardins JDD, lannotti JP. The acromioclavicular capsule as a restraint to posterior translation of the clavicle: a biomechanical analysis. J Shoulder Elbow Surg. 1999;8(2):119-124.

13. Koo TK, Li MY. A guideline of selecting and reporting intraclass correlation coefficients for reliability research. J Chiropr Med. 2016;15(2):155-163.

14. Lee K-W, Debski RE, Chen C-H, Woo SL-Y, Fu FH. Functional evaluation of the ligaments at the acromioclavicular joint during anteroposterior and superoinferior translation. Am J Sports Med. 1997;25(6):858-862.

15. Longo UG, Ciuffreda M, Rizzello G, Mannering N, Maffulli N, Denaro V. Surgical versus conservative management of type III acromioclavicular dislocation: a systematic review. Br Med Bull. 2017;122(1):31-49.

16. Mazzocca AD, Arciero RA, Bicos J. Evaluation and treatment of acromioclavicular joint injuries. Am J Sports Med. 2007;35(2):316-329.

17. Mazzocca AD, Spang JT, Rodriguez RR, et al. Biomechanical and radiographic analysis of partial coracoclavicular ligament injuries. Am J Sports Med. 2008;36(7):1397-1402.

18. Minkus M, Hann C, Scheibel M, Kraus N. Quantification of dynamic posterior translation in modified bilateral Alexander views and correlation with clinical and radiological parameters in patients with acute acromioclavicular joint instability. Arch Orthop Trauma Surg. 2017;137(6):845-852.

19. Motamedi AR, Blevins FT, Willis MC, McNally TP, Shahinpoor M. Biomechanics of the coracoclavicular ligament complex and augmentations used in its repair and reconstruction. Am J Sports Med. 2000;28(3):380-384.

20. Nemec U, Oberleitner G, Nemec SF, et al. Magnetic resonance imaging (MRI) versus radiography of acromioclavicular joint dislocation. AJR Am J Roentgenol. 2011;197(4):968-973.
21. Pogorzelski J, Beitzel K, Ranuccio F, et al. The acutely injured acromioclavicular joint - which imaging modalities should be used for accurate diagnosis? A systematic review. BMC Musculoskelet Disord. 2017;18(1):515.

22. Rahm S, Wieser K, Spross C, Vich M, Gerber C, Meyer DC. Standard axillary radiographs of the shoulder may mimic posterior subluxation of the lateral end of the clavicle. J Orthop Trauma. 2013;27(11): 622-626.

23. Rockwood CA, Green DP. Fractures in Adults. 2nd ed. Lippincott Williams and Wilkins; 1984.

24. Schaefer FK, Schaefer PJ, Brossmann J, Hilgert RE, Heller M, Jahnke T. Experimental and clinical evaluation of acromioclavicular joint structures with new scan orientations in magnetic resonance imaging (MRI). Eur Radiol. 2006;16(7):1488-1493.

25. Shrout PE, Fleiss JL. Intraclass correlations: uses in assessing rater reliability. Psychol Bull. 1979;86(2):420-428.

26. Tamaoki MJ, Lenza M, Matsunaga FT, Belloti JC, Matsumoto MH, Faloppa F. Surgical versus conservative interventions for treating acromioclavicular dislocation of the shoulder in adults. Cochrane Database Syst Rev. 2019;10(10):CD007429.

27. Tauber M, Koller H, Hitzl W, Resch H. Dynamic radiologic evaluation of horizontal instability in acute acromioclavicular joint dislocations. Am J Sports Med. 2010;38(6):1188-1195.

28. Tossy JD, Mead NC, Sigmond HM. Acromioclavicular separations: useful and practical classification for treatment. Clin Orthop Relat Res. 1963;28:111-119.

29. Trainer G, Arciero RA, Mazzocca AD. Practical management of grade III acromioclavicular separations. Clin J Sport Med. 2008;18(2):162-166.

30. Zanca P. Shoulder pain: involvement of the acromioclavicular joint (analysis of 1,000 cases). Am J Roentgenol Radium Ther Nucl Med. 1971;112(3):493-506.

31. Zumstein MA, Schiessl P, Ambuehl B, et al. New quantitative radiographic parameters for vertical and horizontal instability in acromioclavicular joint dislocations. Knee Surg Sports Traumatol Arthrosc. 2017;21(1):215-135.

For reprints and permission queries, please visit SAGE's Web site at http://www.sagepub.com/journalsPermissions.nav. 